\title{
Production of $t Z H$ and $t Z h$ in 2HDM: Prospects for discovery at the LHC
}

\author{
Wei-Shu Hou $\odot$ and Tanmoy Modak \\ Department of Physics, National Taiwan University, Taipei 10617, Taiwan
}

(Received 17 November 2019; accepted 20 January 2020; published 7 February 2020)

\begin{abstract}
We study the discovery potential of the $c g \rightarrow t A \rightarrow t Z H$ process at the LHC, where $A$ and $H$ are $C P$-odd and -even exotic scalars, respectively. The context is the general two Higgs doublet model, where $c g \rightarrow t A$ is induced by the flavor changing neutral Higgs coupling $\rho_{t c}$. We find that the process $c g \rightarrow t A \rightarrow t Z H$ can be discovered for $m_{A} \sim 400 \mathrm{GeV}$, but would likely require high luminosity running of the LHC. Such a discovery would shed light on the mechanism behind the observed baryon asymmetry of the Universe. We also study $c g \rightarrow t A \rightarrow t Z h$, where $h$ is the observed $125 \mathrm{GeV}$ scalar, but find it out of reach at the LHC.
\end{abstract}

DOI: 10.1103/PhysRevD.101.035007

\section{INTRODUCTION}

The discovery of the Higgs boson [1] $h(125)$ at the Large Hadron Collider (LHC) confirms the standard model (SM) as the correct theory at the electroweak scale. As all fermions come in three copies, additional scalars might well exist in nature. In particular, given that $h$ belongs to a weak doublet $\Phi$, extra scalar doublets ought to be searched for. However, the apparent absence of new physics so far at the LHC and the emergent "approximate alignment," i.e., the $h$ boson is found to resemble rather closely the SM Higgs boson, suggest that the extra scalars might be rather heavy. In this so-called decoupling limit [2], where the exotic scalars are multi-TeV in mass, discovery becomes rather difficult even for the High Luminosity LHC (HL-LHC).

By adding just one scalar doublet $\Phi^{\prime}$, the two Higgs doublet model (2HDM) [3] is one of the simplest extensions of the SM. We are interested in sub-TeV exotic scalars $A, H$, and $H^{+}$. The most popular 2HDMs, of interest already before the $h$ boson discovery, are those with a $Z_{2}$ symmetry imposed [3]. The $Z_{2}$ symmetry enforces the up- and downtype quarks to couple to just one scalar doublet, thereby ensuring natural flavor conservation (NFC) [4] and forbids all flavor changing neutral Higgs couplings. But this removes the possibility of any additional Yukawa coupling.

Our context is the general 2HDM (g2HDM), without imposing $Z_{2}$ symmetry. Indeed, approximate alignment can be accommodated $[5,6]$ without taking the decoupling limit, even with $\mathcal{O}(1)$ extra Higgs quartic couplings, clearing the way for sub-TeV $A, H$, and $H^{+}$. In the absence of $Z_{2}$ symmetry, both doublets couple to $u$ - and $d$-type quarks, and

Published by the American Physical Society under the terms of the Creative Commons Attribution 4.0 International license. Further distribution of this work must maintain attribution to the author(s) and the published article's title, journal citation, and DOI. Funded by SCOAP. two separate Yukawa matrices $\lambda_{i j}^{F}=\left(\sqrt{2} m_{i}^{F} / v\right) \delta_{i j}$ (with $v \simeq 246 \mathrm{GeV}$ ) and $\rho_{i j}^{F}$ emerge after diagonalization of the fermion mass matrices. Here, $F$ denotes $u$ - and $d$-type quarks and $e$-type leptons, with the fermion mass and mixing structure and approximate alignment together replacing the NFC condition [5]. The $\lambda$ matrices are real and diagonal, but the $\rho$ matrices are in general nondiagonal and complex. It was pointed out recently that $\mathcal{O}(1) \rho_{t t}$ and $\rho_{t c}$ can drive electroweak baryogenesis (EWBG) rather efficiently $[7,8]$.

If $\rho_{t t}$ and $\rho_{t c}$ are $\mathcal{O}(1)$, one might discover the exotic scalars via the $c g \rightarrow t A / H \rightarrow t t \bar{c}$ process with clean samesign top signature $[9,10]$ (see also Refs. [11-13]), and also with $A / H \rightarrow t \bar{t} t$, i.e., the triple-top process [9]. Induced by only $\rho_{t c}$, the same-sign top process might emerge already with full Run-2 data. On the other hand, the more exquisite triple-top process, which depends on both $\rho_{t t}$ and $\rho_{t c}$ couplings, may require the inclusion of Run 3 data to show any indication. But if $\rho_{t t}$ is negligibly small, the triple-top discovery would not be possible. In this paper we consider the case where $\rho_{t c}$ is $\mathcal{O}(1)$ but $\rho_{t t}$ is tiny, where another novel discovery mode would be $c g \rightarrow t A \rightarrow t Z H$ (charge conjugate process always implied) for $m_{A}>$ $m_{Z}+m_{H}$. With no dilution from $A \rightarrow t \bar{t}$, the process can provide an additional discovery mode that is complementary to Refs. $[9,10]$, and provide additional information on $\rho_{t c}$ driven EWBG.

The $c g \rightarrow t A \rightarrow t Z H$ process can be searched for in the inclusive $\quad p p \rightarrow t A+X \rightarrow t Z H+X$ process, with $Z \rightarrow \ell^{+} \ell^{-}, H \rightarrow \bar{t} c+t \bar{c}$, and at least one top decaying semileptonically. We call this the $t Z H$ process, the observation of which has another intriguing impact. It has been shown that the $A \rightarrow Z H$ decay can provide a smoking gun signature for the strongly first order electroweak phase transition (EWPT) which might have occurred in the early Universe [14-16]. A strongly first order EWPT is needed for the out of equilibrium condition that is required for 
successful EWBG [17]. Realizing the importance [18], indeed both ATLAS and CMS have pursued the $g g \rightarrow A \rightarrow$ $Z H$ search $[19,20]$. However, if $\rho_{t t}$ is tiny, $g g \rightarrow A$ vanishes, and the $t Z H$ process is a unique probe of the strongly first order EWPT mechanism, as well as the $\rho_{t c}$ driven EWBG scenario.

For completeness, we also study the prospect for the $c g \rightarrow t A \rightarrow t Z h$ process. The process is also induced by $\rho_{t c}$, but would depend on $\cos \gamma$, the $h-H$ mixing angle. The process can be searched for via $p p \rightarrow t A+X \rightarrow t Z h+X$, with $t \rightarrow b \ell^{+} \nu_{\ell}, Z \rightarrow \ell^{+} \ell^{-}$, and $h \rightarrow b \bar{b}$, which we call the $t Z h$ process. It provides another complementary probe of the $\rho_{t c}$ driven EWBG scenario, as well as the $c_{\gamma}$ mixing angle if $\rho_{t t}$ is rather small.

In the following, we first discuss the framework in Sec. II, followed by the parameter space and discovery potential of the $t Z H$ process in Sec. III. Section IV is dedicated to the $t Z h$ process, and we summarize our results with some discussion in Sec. V.

\section{FRAMEWORK}

The scalars $h, H, A$, and $H^{+}$couple to fermions by [21]

$$
\begin{aligned}
\mathcal{L}= & -\frac{1}{\sqrt{2}} \sum_{F=U, D, L^{\prime}} \bar{F}_{i}\left[\left(-\lambda_{i j}^{F} s_{\gamma}+\rho_{i j}^{F} c_{\gamma}\right) h\right. \\
& \left.+\left(\lambda_{i j}^{F} c_{\gamma}+\rho_{i j}^{F} s_{\gamma}\right) H-i \operatorname{sgn}\left(Q_{F}\right) \rho_{i j}^{F} A\right] R F_{j} \\
& -\bar{U}_{i}\left[\left(V \rho^{D}\right)_{i j} R-\left(\rho^{U_{\dagger}} V\right)_{i j} L\right] D_{j} H^{+} \\
& -\bar{\nu}_{i} \rho_{i j}^{L} R L_{j}^{\prime} H^{+}+\text {H.c. },
\end{aligned}
$$

where $L, R \equiv\left(1 \mp \gamma_{5}\right) / 2, i, j=1,2,3$ are generation indices, $V$ is Cabibbo-Kobayashi-Maskawa matrix, $c_{\gamma}=$ $\cos \gamma$ is the $h-H$ mixing angle between $C P$-even scalars, and $U=(u, c, t), D=(d, s, b), L^{\prime}=(e, \mu, \tau)$ and $\nu=$ $\left(\nu_{e}, \nu_{\mu}, \nu_{\tau}\right)$ are vectors in flavor space. The matrices $\lambda_{i j}^{F}\left(=\sqrt{2} m_{i}^{F} / v\right)$ are real and diagonal, whereas $\rho_{i j}^{F}$ are in general complex and nondiagonal.

In the Higgs basis, the most general $C P$-conserving two Higgs doublet potential can be written as $[5,21]$

$$
\begin{aligned}
V\left(\Phi, \Phi^{\prime}\right)= & \mu_{11}^{2}|\Phi|^{2}+\mu_{22}^{2}\left|\Phi^{\prime}\right|^{2}-\left(\mu_{12}^{2} \Phi^{\dagger} \Phi^{\prime}+\text { H.c. }\right) \\
& +\frac{\eta_{1}}{2}|\Phi|^{4}+\frac{\eta_{2}}{2}\left|\Phi^{\prime}\right|^{4}+\eta_{3}|\Phi|^{2}\left|\Phi^{\prime}\right|^{2}+\eta_{4}\left|\Phi^{\dagger} \Phi^{\prime}\right|^{2} \\
& +\left[\frac{\eta_{5}}{2}\left(\Phi^{\dagger} \Phi^{\prime}\right)^{2}+\left(\eta_{6}|\Phi|^{2}+\eta_{7}\left|\Phi^{\prime}\right|^{2}\right) \Phi^{\dagger} \Phi^{\prime}+\text { H.c. }\right],
\end{aligned}
$$

where the vacuum expectation value $v$ arises from the doublet $\Phi$ via the minimization condition $\mu_{11}^{2}=-\frac{1}{2} \eta_{1} v^{2}$, while $\left\langle\Phi^{\prime}\right\rangle=0$ (hence $\mu_{22}^{2}>0$ ), and $\eta_{i}$ s are quartic couplings. Here we follow the notation of Ref. [5]. A second minimization condition, $\mu_{12}^{2}=\frac{1}{2} \eta_{6} v^{2}$, removes $\mu_{12}^{2}$, and the total number of parameters are reduced to 9 [5].
Two relations [5] arise for the mixing angle $\gamma$ when diagonalizing the mass-squared matrix for $h, H$,

$$
c_{\gamma}^{2}=\frac{\eta_{1} v^{2}-m_{h}^{2}}{m_{H}^{2}-m_{h}^{2}}, \quad \sin 2 \gamma=\frac{2 \eta_{6} v^{2}}{m_{H}^{2}-m_{h}^{2}} .
$$

The alignment limit, $c_{\gamma} \rightarrow 0$, is reached for $\eta_{6} \rightarrow 0$ [5], hence $m_{h}^{2} \rightarrow \eta_{1} v^{2}$, or via decoupling [2], i.e., $m_{H}^{2} \gg v^{2}$. But for small but not infinitesimal $c_{\gamma}$, one has $c_{\gamma} \simeq$ $\left|\eta_{6}\right| v^{2} /\left(m_{H}^{2}-m_{h}^{2}\right)$. This is the so-called approximate alignment [5]; i.e., small $c_{\gamma}$ values can be attained with $\eta_{6}, \eta_{1}>m_{h}^{2} / v^{2}$. The scalar masses can be expressed in terms of the parameters in Eq. (2),

$$
\begin{aligned}
m_{h, H}^{2}=\frac{1}{2}[ & m_{A}^{2}+\left(\eta_{1}+\eta_{5}\right) v^{2} \\
& \left.\mp \sqrt{\left(m_{A}^{2}+\left(\eta_{5}-\eta_{1}\right) v^{2}\right)^{2}+4 \eta_{6}^{2} v^{4}}\right],
\end{aligned}
$$

$$
m_{A}^{2}=\frac{1}{2}\left(\eta_{3}+\eta_{4}-\eta_{5}\right) v^{2}+\mu_{22}^{2},
$$

$$
m_{H^{ \pm}}^{2}=\frac{1}{2} \eta_{3} v^{2}+\mu_{22}^{2}
$$

The processes of interest are $c g \rightarrow t A \rightarrow t Z H$ and $t Z h$, where $c g \rightarrow t A$ is induced by $\rho_{t c}$, but the $A \rightarrow Z H, Z h$ decays via the gauge couplings $[3,22]$

$$
\frac{g_{2}}{2 c_{W}} Z_{\mu}\left[c_{\gamma}\left(h \partial^{\mu} A-A \partial^{\mu} h\right)-s_{\gamma}\left(H \partial^{\mu} A-A \partial^{\mu} H\right)\right],
$$

with $c_{W}$ being the Weinberg angle and $g_{2}$ the $S U(2)_{L}$ gauge coupling. We see from Eq. (7) that $A \rightarrow Z H$ is proportional to $s_{\gamma}$, while $A \rightarrow Z h$ is proportional to $c_{\gamma}$. The coupling $\rho_{c t}$ can also generate $c g \rightarrow t A$, but it is very stringently constrained by flavor physics [23]. We set $\rho_{c t}$ to 0 throughout the paper for simplicity.

For nonzero $\rho_{t c}$, we remark that the discovery at the LHC, if at all, would first occur through the $c g \rightarrow t A \rightarrow t t \bar{c}$ process $[9,10]$. For $m_{A}<2 m_{t}$, if other $\rho_{i j} \mathrm{~s}$ are small, $c g \rightarrow$ $t A \rightarrow t Z H$ could be the only process to emerge after $c g \rightarrow t A \rightarrow t t \bar{c}$. For $m_{A}>2 m_{t}, c g \rightarrow t A \rightarrow t t \bar{c}$ would in general be accompanied by the $c g \rightarrow t A \rightarrow t t \bar{t}$ process [9], unless $\rho_{t t}$ is negligibly small, which we assume. We focus on $t \rightarrow b \ell^{+} \nu_{\ell}, H \rightarrow t \bar{c}+\bar{t} c$, and $Z \rightarrow \ell^{+} \ell^{-}$decays, with the top quark from $H$ decay also decaying semileptonically. Thus, following a possible $c g \rightarrow t A \rightarrow t t \bar{c}$ discovery, $c g \rightarrow$ $t A \rightarrow t Z H$ could be the only process that might provide a complementary probe of the $\rho_{t c}$ driven EWBG, even for approximate alignment (i.e., small $c_{\gamma}$ ) [24]. In the following, we assume $\rho_{t c}$ is the only nonzero coupling and set all other couplings to 0 . Their impact, however, is discussed later in the paper. 
The prospect for $c g \rightarrow t A \rightarrow t Z h$ closely depends on the mixing angle $c_{\gamma}$, vanishing for $c_{\gamma} \rightarrow 0$. For large $\rho_{t t}, g g \rightarrow$ $A \rightarrow Z h$ [25] probes $c_{\gamma}$. For negligibly small $\rho_{t t}$, the process $c g \rightarrow t A \rightarrow t Z h$ can provide unique probe of $c_{\gamma}$. We focus on $t \rightarrow b \ell^{+} \nu_{\ell}, h \rightarrow b \bar{b}$, and $Z \rightarrow \ell^{+} \ell^{-}$.

\section{THE $t Z H$ PROCESS}

In this section we analyze the discovery potential of the $t \mathrm{ZH}$ process at the LHC. We first look at the relevant constraints on the parameter space, then find the discovery potential at $\sqrt{s}=14 \mathrm{TeV}$. For simplicity, we assume all $\rho_{i j}=0$ except $\rho_{t c}$. However, the impact of other $\rho_{i j} \mathrm{~s}$ is discussed later in the paper. To simplify further, we set $c_{\gamma}=0$ throughout this section.

\section{A. Parameter space}

Let us find the available parameter space for the $t \mathrm{ZH}$ process. We first focus on the mass spectrum of the extra scalars $A, H$, and $H^{+}$. The process requires $A$ heavier than $H$ by at least $m_{Z}$. To find whether such a mass spectrum exists, the dynamical parameters in Eq. (2) need to satisfy positivity, perturbativity, and tree-level unitarity conditions, for which we utilize 2HDMC [26]. We first express the quartic couplings $\eta_{1}, \eta_{3-6}$ in terms of $[5,21] \mu_{22}, m_{h}, m_{H}$, $m_{A}, m_{H^{ \pm}}$, all normalized to $v$, and the mixing angle $\gamma$,

$$
\begin{aligned}
& \eta_{1}=\frac{m_{h}^{2} s_{\gamma}^{2}+m_{H}^{2} c_{\gamma}^{2}}{v^{2}}, \\
& \eta_{3}=\frac{2\left(m_{H^{ \pm}}^{2}-\mu_{22}^{2}\right)}{v^{2}}, \\
& \eta_{4}=\frac{m_{h}^{2} c_{\gamma}^{2}+m_{H}^{2} s_{\gamma}^{2}-2 m_{H^{ \pm}}^{2}+m_{A}^{2}}{v^{2}}, \\
& \eta_{5}=\frac{m_{H}^{2} s_{\gamma}^{2}+m_{h}^{2} c_{\gamma}^{2}-m_{A}^{2}}{v^{2}}, \\
& \eta_{6}=\frac{\left(m_{h}^{2}-m_{H}^{2}\right)\left(-s_{\gamma}\right) c_{\gamma}}{v^{2}} .
\end{aligned}
$$

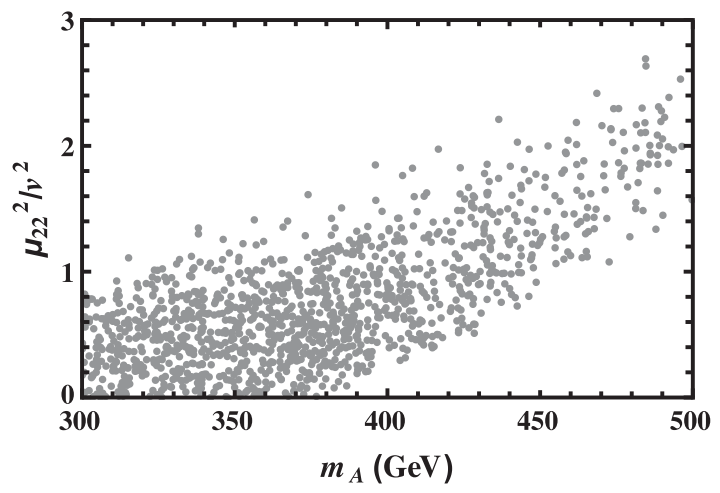

The quartic couplings $\eta_{2}$ and $\eta_{7}$ do not enter scalar masses; nor does the mixing angle $\gamma$. Therefore, in our analysis we take $v, m_{h}$, and $\gamma, m_{A}, m_{H}, m_{H^{ \pm}}, \mu_{22}, \eta_{2}, \eta_{7}$ as the phenomenological parameters.

To save computation time, we randomly generate these parameters in the following ranges: $\eta_{2} \in[0,3]$, $\eta_{7} \in[-3,3], \mu_{22} \in[0,1000] \mathrm{GeV}, m_{A} \in[300,500] \mathrm{GeV}$, $m_{H} \in\left[200, m_{A}-m_{Z}\right] \mathrm{GeV}, m_{H^{ \pm}} \in[300,500] \mathrm{GeV}$, while satisfying $m_{h}=125 \mathrm{GeV}$. Note that since the $c g \rightarrow t A \rightarrow$ $t \mathrm{ZH}$ process depends only on $s_{\gamma}$, for simplicity we take $c_{\gamma}=0$ in this section. To simplify further, we demand $m_{A}<m_{H^{ \pm}}+m_{W}$ to forbid the $A \rightarrow H^{ \pm} W^{\mp}$ decay. We then pass the randomly generated parameters to $2 \mathrm{HDMC}$ for scanning, which uses [26] $m_{H^{ \pm}}$and $\Lambda_{1-7}$ as input parameters in the Higgs basis with $v$ as an implicit parameter. To match the 2 HDMC convention, we identify $\eta_{1-7}$ as $\Lambda_{1-7}$ and take $-\pi / 2 \leq \gamma \leq \pi / 2$, and $\eta_{2}$ needs to be greater than 0 as required by positivity, along with other more involved conditions in 2HDMC. In addition, we further conservatively demand all $\left|\eta_{i}\right| \leq 3$.

One also has to consider the stringent oblique $T$ parameter [27] constraint, which restricts the scalar masses $m_{A}, m_{H}$, and $m_{H^{+}}$[28,29], and therefore the quartic couplings $\eta_{i}$ s. We use the $T$ parameter expression given in Ref. [28] and check that the points that passed positivity, unitarity, and perturbativity conditions in 2HDMC also satisfy the $T$ parameter constraint within $2 \sigma$ error [30]. These final points together are called "scan points," which are plotted as gray dots in Fig. 1 in the $\mu_{22}^{2} / v^{2}$ and $\mid \eta_{3}+$ $\eta_{4}-\eta_{5} \mid$ vs $m_{A}$ planes. The figure illustrates that there exists finite parameter space for $300 \mathrm{GeV} \lesssim m_{A} \lesssim 500 \mathrm{GeV}$, which can facilitate $A \rightarrow Z H$ decay. In general, heavier $m_{A}$ are possible, but the discovery potential diminishes with the rapid falloff in parton luminosity. From the scan points in Fig. 1, we choose three benchmark points (BPs) for our analysis, which are summarized in Table I.

The coupling $\rho_{t c}$ is constrained by both LHC search and flavor physics. As we assume $c_{\gamma}=0$ throughout this section, the most stringent limit arises from the CMS search for four-top production [31], where the CRW region, i.e., control region for $t \bar{t} W$ background, gives the most

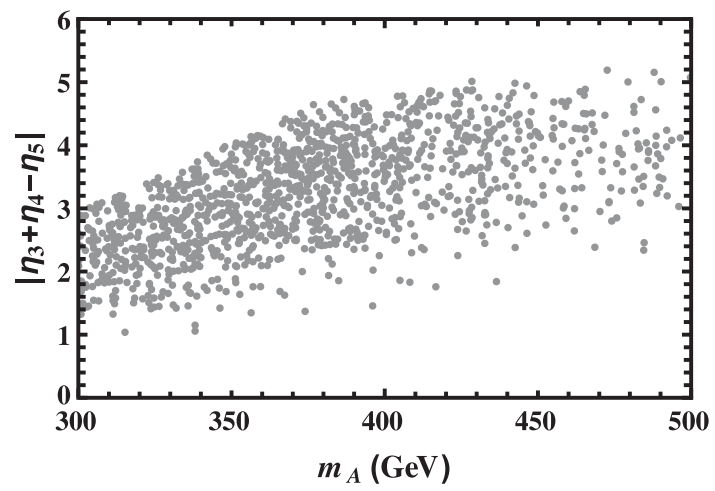

FIG. 1. The scanned points plotted in the $\mu_{22}^{2} / v^{2}$ vs $m_{A}$ (left) and $\left|\eta_{3}+\eta_{4}-\eta_{5}\right|$ vs $m_{A}$ (right) plane. 
TABLE I. Parameter values for the three benchmark points. See the text for details.

\begin{tabular}{lcccccccccccc}
\hline \hline BP & $\eta_{1}$ & $\eta_{2}$ & $\eta_{3}$ & \multicolumn{1}{c}{$\eta_{4}$} & \multicolumn{1}{c}{$\eta_{5}$} & \multicolumn{1}{c}{$\eta_{345}$} & $\eta_{6}$ & \multicolumn{1}{c}{$\eta_{7}$} & $m_{H^{ \pm}}(\mathrm{GeV})$ & $m_{A}(\mathrm{GeV})$ & $m_{H}$ & $\frac{\mu_{22}^{2}}{v^{2}}(\mathrm{GeV})$ \\
\hline$a$ & 0.258 & 2.133 & 2.87 & -0.569 & -1.194 & 1.107 & 0 & -0.791 & 310 & 339 & 207 & 0.15 \\
$b$ & 0.258 & 1.366 & 2.718 & -0.733 & -1.97 & 0.015 & 0 & -0.252 & 354 & 404 & 208 & 0.71 \\
$c$ & 0.258 & 2.432 & 2.67 & -0.652 & -2.21 & -0.192 & 0 & 0.091 & 393 & 449 & 260 & 1.21 \\
\hline \hline
\end{tabular}

relevant constraint. For nonzero $\rho_{t c}$, the process $c g \rightarrow$ $t H / t A \rightarrow t t \bar{c}$ with same-sign top (same sign leptons plus jets) contributes abundantly to the CRW region, resulting in stringent constraint on $\rho_{t c}$. There is, however, a subtlety. The $c g \rightarrow t H \rightarrow t t \bar{c}$ and $c g \rightarrow t A \rightarrow t t \bar{c}$ processes cancel each other exactly by destructive interference, if the masses and widths of $H$ and $A$ are the same $[9,10]$. This cancellation diminishes [10] when the $m_{A}-m_{H}$ mass splitting is larger than the respective widths, which is the case for all three BPs, where $m_{A}-m_{H}$ is more than $100 \mathrm{GeV}$. We refrain from a detailed discussion on the extraction procedure for this constraint, but refer the reader to Refs. [10,32]. Following the procedure in Ref. [10] and utilizing the CRW region of Ref. [31], we find the $95 \%$ C.L. upper limits on $\rho_{t c}$ are $0.4,0.5,0.45$ for the $\mathrm{BP} a, \mathrm{BP} b$, and $\mathrm{BP} c$, respectively.

The constraints from $\mathcal{B}\left(B \rightarrow X_{s} \gamma\right)$ and $B_{q}$ mixing $(q=d, s)$ on $\rho_{t c}$ should also be considered, where $\rho_{t c}$ enters via $\mathrm{H}^{+}$coupling in the charm loop [33,34]. For example, reinterpreting the result of Ref. [33], one finds that $\left|\rho_{t c}\right| \gtrsim 1$ is excluded for $m_{H^{+}}=300 \mathrm{GeV}$ from $B_{s}$ mixing, the ballpark mass range for $m_{H^{+}}$for all three BPs. The constraints are weaker than those from the CRW region. At this point we remark that lighter $m_{A}, m_{H}$, and, $m_{H^{ \pm}}$compared to the three BPs are also possible, but the constraints on $\left|\rho_{t c}\right|$ from the CRW region, $\mathcal{B}\left(B \rightarrow X_{s} \gamma\right)$ and $B_{q}$ mixing, would be more severe.

For nonvanishing $c_{\gamma}, \rho_{t c}$ receives further constraints from $\mathcal{B}(t \rightarrow c h)$ measurement. Although we set $c_{\gamma}=0$ in this section, let us briefly discuss this constraint. Both ATLAS and CMS have searched for $t \rightarrow c h$ decay and set 95\% C.L. upper limits. The latest ATLAS result is based on $36.1 \mathrm{fb}^{-1}$ data at $13 \mathrm{TeV}$, setting the limit $\mathcal{B}(t \rightarrow c h)<1.1 \times 10^{-3}$ [35], while the CMS limit is $\mathcal{B}(t \rightarrow c h)<4.7 \times 10^{-3}$ [36], based on $35.9 \mathrm{fb}^{-1}$. The ATLAS constraint on $\mathcal{B}(t \rightarrow c h)$ [35] is illustrated in the $\rho_{t c}-c_{\gamma}$ plane as the purple shaded region in Fig. 2, where we do not display the weaker CMS limit. Taking $c_{\gamma}=0.2$, for example, one gets the upper limit of $\left|\rho_{t c}\right| \lesssim 0.5$ at $95 \%$ C.L. [37], but the limit weakens for smaller $c_{\gamma}$.

Under the assumptions made, there are only two decay modes, $A \rightarrow t \bar{c}+\bar{t} c$ and $A \rightarrow Z H$, for all three benchmark points. These branching ratios are summarized in Table II, while $\mathcal{B}(H \rightarrow t \bar{c}+\bar{t} c)=1$. We note that for fixed $m_{H}$, $\mathcal{B}(A \rightarrow Z H)$ is larger for heavier $m_{A}$; hence $\mathcal{B}(A \rightarrow Z H)$ of $\mathrm{BP} a$ is smaller than that of $\mathrm{BP} b$. The total decay widths of
$A(H)$ for the three BPs respectively are $2.91(0.18), 9.78$ (0.29), and, 9.65 (0.98) GeV.

\section{B. Collider signature}

We analyze the discovery prospects for $c g \rightarrow t A \rightarrow t Z H$ at the LHC with $\sqrt{s}=14 \mathrm{TeV}$. The process can be searched for via $p p \rightarrow t A+X \rightarrow t Z H+X \rightarrow t Z(t \bar{c}+\bar{t} c)+X$, with $Z \rightarrow \ell^{+} \ell^{-}$and at least one of the final state top quarks decaying semileptonically. $Z \rightarrow \tau^{+} \tau^{-}, \nu \bar{\nu}$ decays are also possible, but we do not find them as promising. The dominant backgrounds for the $t Z H$ process arise from $t \bar{t} Z$ and $W Z+$ jets processes, while $t W Z$, four-top quarks (4t), $t \bar{t} h, t t \bar{W}$, and $t Z+$ jets are subdominant. Minor contributions come from $3 t+$ jets and $3 t+W$ jets.

In order to find the discovery potential of the three benchmark points, we generate background and signal event samples at leading order (LO) by Monte Carlo event generator MadGraph5_aMC@NLO [38] with the parton distribution function (PDF) set NN23LO1 [39] at $\sqrt{s}=14 \mathrm{TeV}$. The event samples are then interfaced with PYTHIA 6.4 [40] for showering and hadronization, and finally fed into Delphes 3.4.0 [41] to incorporate detector effects. We have generated the matrix elements (ME) of signal and all backgrounds except for the $W Z+$ jets with up to one additional jet in the final state, followed by ME and parton shower merging with the MLM matching scheme [42,43]. We considered two additional jets for $\mathrm{ME}$ and parton shower merging for $W Z+$ jets background. We have not included backgrounds arising from the nonprompt and fake sources, as they are not properly modeled in Monte Carlo

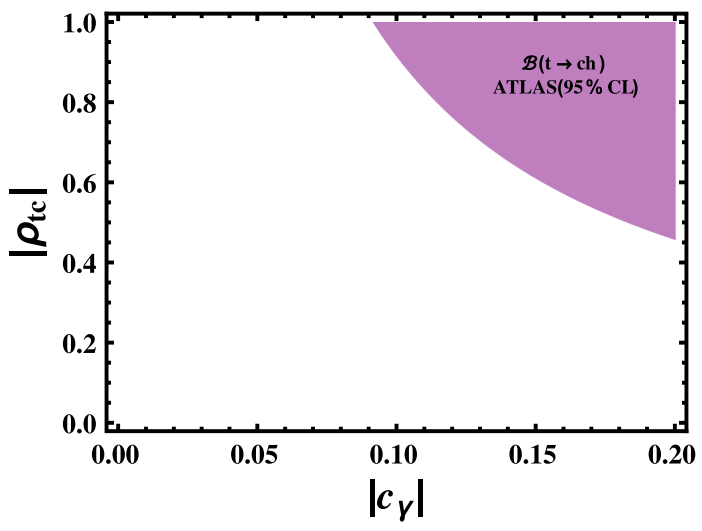

FIG. 2. Constraint from $\mathcal{B}(t \rightarrow c h)$ measurement in $\rho_{t c}$ vs $c_{\gamma}$. 
TABLE II. Branching ratios for the benchmark points.

\begin{tabular}{llcc}
\hline \hline BP & $\rho_{t c}$ & $\mathcal{B}(A \rightarrow t \bar{c}+\bar{c} t)$ & $\mathcal{B}(A \rightarrow Z H)$ \\
\hline$a$ & 0.4 & 0.61 & 0.39 \\
$b$ & 0.5 & 0.41 & 0.59 \\
$c$ & 0.45 & 0.41 & 0.59 \\
\hline \hline
\end{tabular}

simulations, and usually require data to make estimates. Here we have incorporated the default ATLAS-based detector card available within the Delphes framework. The effective model is implemented in FeynRules [44].

The dominant $t \bar{t} Z$ cross section at $\mathrm{LO}$ is normalized to the next-to-leading order (NLO) by the $K$ factor 1.56 [45]. The $W Z+$ jets background is adjusted to next-to-next-toleading order cross section by a factor 2.07 [46]. Furthermore, the LO $\bar{t} Z+$ jets, $t \bar{t} h, 4 t$ and $t \bar{t} W^{-}\left(t \bar{t} W^{+}\right)$ cross sections are adjusted to NLO by $K$ factors 1.44 [38], 1.27 [47], 2.04 [38], and 1.35 (1.27) [48], respectively, while the cross sections for $3 t+$ jets, $3 t+W$ jets and $t W Z$ are kept at LO. For simplicity, the QCD correction factors for the $t Z j$ and $W^{+} Z+$ jets processes are assumed to be the same as their respective charge conjugate processes. The signal cross sections for all three BPs are kept at LO.

Let us discuss the event selection criteria for the $t Z H$ process. Each event should contain at least three charged leptons $(e$ and $\mu$ ), at least three jets with at least two $b$ tagged, and missing transverse energy $\left(E_{T}^{\text {miss }}\right)$. The transverse momenta, $p_{T}$, of the leading charged lepton should be $>25 \mathrm{GeV}$, while the other two leptons should have $p_{T}>20 \mathrm{GeV}$. The minimum transverse energy $E_{T}^{\text {miss }}$ needs to be $>35 \mathrm{GeV}$. All three jets are required to have $p_{T}>20 \mathrm{GeV}$. The absolute value of pseudorapidity, $|\eta|$, of the three leading leptons and three jets (which includes two $b$-tagged jets) should be $<2.5$. The separation $\Delta R$ between any two leptons, any two jets, and any jet and lepton should be $>0.4$. The jets are reconstructed by utilizing anti- $k_{T}$ algorithm with radius parameter $R=0.6$.

The invariant mass of the two opposite-charge, sameflavor leptons, $m_{\ell^{+} \ell^{-}}$, is required to be within the $Z$ boson mass window $76<m_{\ell^{+} \ell^{-}}<100 \mathrm{GeV}$. As there are at least three charged leptons in the event, with two coming from $Z$ decay and one from one of the $t$ quark decays, there are at least two combinations of $m_{\ell^{+} \ell^{-}}$. We identify the pair having the invariant mass $m_{\ell^{+} \ell^{-}}$closest to $m_{Z}$ as the one coming from $Z$ decay, and then impose the $m_{\ell^{+} \ell^{-}}$mass cut. We finally veto events for $E_{T}^{\text {miss }}>150,250$, and $270 \mathrm{GeV}$ for $\mathrm{BP} a, \mathrm{BP} b$, and $\mathrm{BP} c$, respectively. The $E_{T}^{\text {miss }}$ veto helps reduce the dominant $t \bar{t} Z$ background for all three BPs.

The normalized $m_{\ell^{+} \ell^{-}}$and $E_{T}^{\text {miss }}$ distributions before any selection cuts (with minimal default cuts during event generation in MadGraph5_aMC@NLO) for the three BPs and backgrounds are plotted in Fig. 3.

In this exploratory study, for simplicity we have not optimized the selection cuts such as $m_{\ell^{+} \ell^{-}}$and $E_{T}^{\text {miss }}$ for our BPs. The background cross sections after selection cuts are summarized in Table III for all three BPs. In Table IV we give signal cross sections and the corresponding significance for the integrated luminosities $\mathcal{L}=600$ and $3000 \mathrm{fb}^{-1}$. The statistical significances in Table IV are determined by using $\mathcal{Z}=\sqrt{2[(S+B) \ln (1+S / B)-S]}$ [49], where $S$ and $B$ are the number of signal and background events after selection.

We find that the significances can reach up to $\sim 1.5 \sigma$, $2.7 \sigma$, and $2.1 \sigma$ for $\mathrm{BP} a, \mathrm{BP} b$, and $\mathrm{BP} c$, respectively, for $600 \mathrm{fb}^{-1}$. With the full HL-LHC data set (i.e., $3000 \mathrm{fb}^{-1}$ integrated luminosity) one can have $\sim 3.4 \sigma, 6 \sigma$, and $4.8 \sigma$ for the BPs, respectively. With moderate $S / B \sim 10 \%$ for the three BPs, these significances illustrates that discovery is possible for $m_{A} \sim 400 \mathrm{GeV}$, while evidence is possible for $m_{A} \sim 350 \mathrm{GeV}$. That significance is lower for lighter $m_{A}$ should not be surprising, since $\mathcal{B}(A \rightarrow Z H)$ is lower for
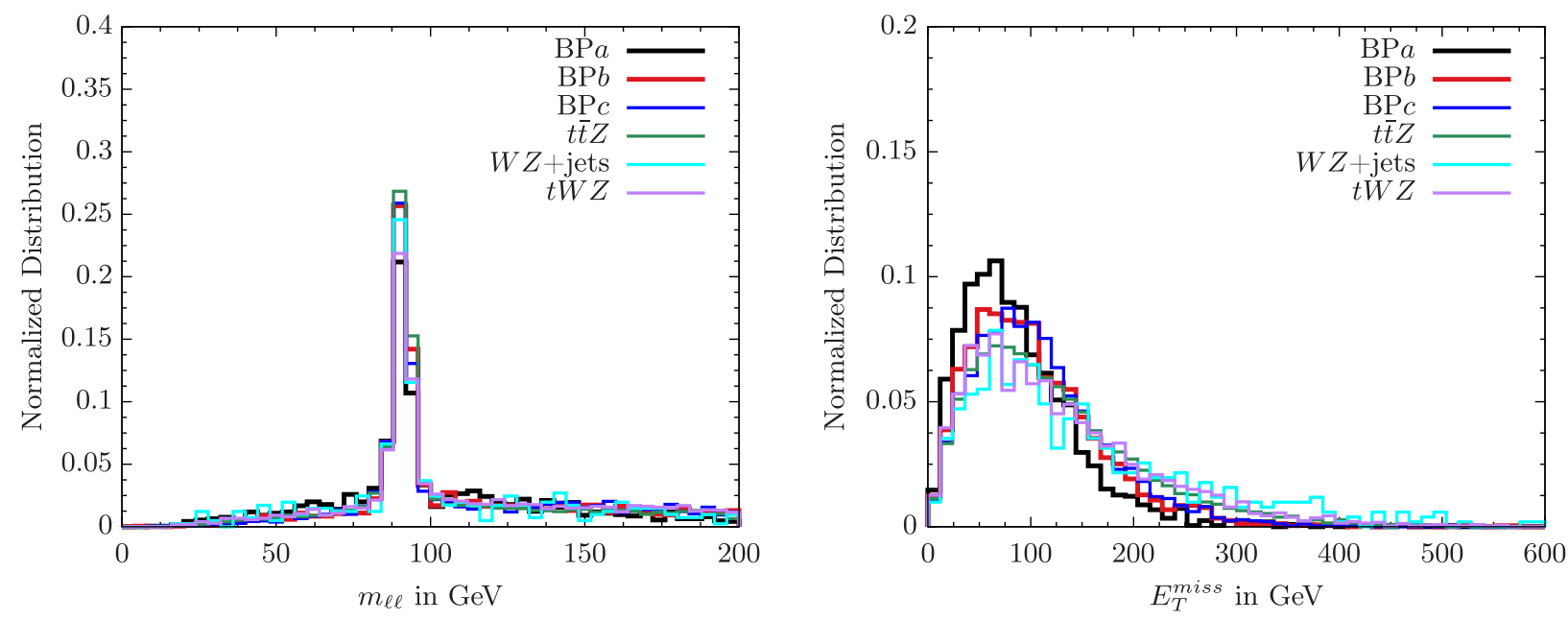

FIG. 3. The normalized $m_{\ell^{+} \ell^{-}}$(left) and $E_{T}^{\text {miss }}$ (right) distributions for the signal and background processes. 
TABLE III. Background cross sections (in fb) for the $t Z H$ process after selection cuts at $\sqrt{s}=14$ TeV LHC. The subdominant $3 t+$ jets, $3 t+W$ are added together as others in the second to last column, while the last column is the total background.

\begin{tabular}{lccccccccc}
\hline \hline BP & $t \bar{t} Z$ & $W Z+$ jets & $t W Z$ & $4 t$ & $t \bar{t} h$ & $t t \bar{W}$ & $t Z+$ jets & Others & Total background \\
\hline$a$ & 0.655 & 0.077 & 0.025 & 0.003 & 0.003 & 0.003 & 0.006 & 0.0001 & 0.772 \\
$b$ & 0.902 & 0.11 & 0.035 & 0.004 & 0.004 & 0.004 & 0.007 & 0.0002 & 1.066 \\
$c$ & 0.925 & 0.112 & 0.036 & 0.005 & 0.004 & 0.004 & 0.007 & 0.0002 & 1.093 \\
\hline \hline
\end{tabular}

TABLE IV. $t Z H$ signal cross sections and significances after selection cuts for the three benchmark points.

\begin{tabular}{lcc}
\hline \hline BP & Signal $(\mathrm{fb})$ & Significance $(\mathcal{Z}) 600(3000) \mathrm{fb}^{-1}$ \\
\hline$a$ & 0.055 & $1.5(3.4)$ \\
$b$ & 0.115 & $2.7(6.0)$ \\
$c$ & 0.092 & $2.1(4.8)$ \\
\hline \hline
\end{tabular}

$\mathrm{BP} a$ than $\mathrm{BP} b$ and $\mathrm{BP} c$. For heavier $m_{A}$ in $\mathrm{BP} b$ and $\mathrm{BP} c$, such enhancement in branching ratios can compensate the lower $c g \rightarrow t A$ production cross section due to a fall in parton luminosity. Our results illustrate that $\sim 2 \sigma$ hint is possible for $m_{A} \sim 400 \mathrm{GeV}$ at Run $3\left(300 \mathrm{fb}^{-1}\right)$, but discovery would require the HL-LHC. The achievable significances depend mildly on the applied $E_{T}^{\text {miss }}$ veto; e.g., if we apply the same $E_{T}^{\text {miss }}$ veto that is chosen for $\mathrm{BP} a$ to $\mathrm{BP} b$ and $\mathrm{BP} c$, the significances of the latter two BPs would drop by $\sim 10 \%$ and $\sim 17 \%$, respectively. However, rejecting events with $E_{T}^{\text {miss }}>250 \mathrm{GeV}$ would enhance the significance for $\mathrm{BP} a$ by $\sim 18 \%$ but reduce by $\sim 8 \%$ for $\mathrm{BP} c$, while keeping the significance for $\mathrm{BP} b$ unchanged. We remark that in our exploratory analysis we have not optimized the $E_{T}^{\text {miss }}$ cut and leave a more detailed analysis for future.

So far we have set all $\rho_{i j}=0$ except $\rho_{t c}$. Before closing this subsection, let us briefly discuss the impact of other $\rho_{i j}$ couplings. If $\rho_{i j}$ follows similar flavor organization structure as in SM, $\rho_{t t}$ could be $\mathcal{O}\left(\lambda_{t}\right), \rho_{b b} \sim \lambda_{b}$, and $\rho_{\tau \tau} \sim \lambda_{\tau}$. In general, the presence of other $\rho_{i j}$ s opens up further decay modes of $A$ and $H$, which in turn dilutes $\mathcal{B}(A \rightarrow Z H)$, and hence the discovery potential of the $t Z H$ process. For example, if $\rho_{t t}=\lambda_{t}(0.5)$, the achievable significances for $\mathrm{BP} b$ and $\mathrm{BP} c$ with full HL-LHC data set are reduced to $\sim 2.7 \sigma(4.6 \sigma)$ and $1.7 \sigma(3.3 \sigma)$, respectively, due to nonzero $\mathcal{B}(A \rightarrow t \bar{t})$. The significance of $\mathrm{BP} a$ would remain unchanged as $m_{A}<2 m_{t}$. Impact of other $\rho_{i j}$ couplings is significantly milder than $\rho_{t t}$. For example, for $\rho_{b b} \sim \lambda_{b}$ and $\rho_{\tau \tau} \sim \lambda_{\tau}$, the significance in Table IV remains practically the same.

Complex $\rho_{t t}$ provides a generally more robust mechanism for EWBG $[7,8]$. Having nonzero $\rho_{t t}$ motivates the conventional $g g \rightarrow H \rightarrow t \bar{t}$ scalar resonance search, or $g g \rightarrow H t \bar{t} \rightarrow t \bar{t} t \bar{t}$ [50], i.e., four-top search. The former process suffers from large interference [51] with the overwhelming $g g \rightarrow t \bar{t}$ background, leading to a peak-dip signature that makes detection difficult, but recent searches by ATLAS [52] and CMS [53] find some sensitivity. See Ref. [32] for a recent discussion in the g2HDM context. Presence of both $\rho_{t c}$ and $\rho_{t t}$ can induce $g g \rightarrow A / H \rightarrow t \bar{c}$ [23] and $c g \rightarrow t A / t H \rightarrow t t \bar{t}$ processes [9], which can also be observable at the LHC, but the former may suffer from $t+j$ mass resolution, which could be close to $200 \mathrm{GeV}$ [54].

\section{THE $t$ Zh PROCESS}

We discuss the prospect of $t Z h$ process, i.e., $p p \rightarrow$ $t A+X \rightarrow t Z h+X$, with $t \rightarrow b \ell^{+} \nu_{\ell}, Z \rightarrow \ell^{+} \ell^{-}(\ell=e, \mu)$, and $h \rightarrow b \bar{b}$. The process depends heavily on the mixing angle $c_{\gamma}$, as well as $\rho_{t c}$. In addition to the constraint from the CMS CRW region [31], it also receives constraint from ATLAS $\mathcal{B}(t \rightarrow c h)$ [35]. Indeed, larger $c_{\gamma}$ enhances $\mathcal{B}(A \rightarrow Z h)$, but $c g \rightarrow t A$ production is balanced by the stronger constraint on $\rho_{t c}$, as can be seen from Fig. 2. The process is further plagued by tiny $\mathcal{B}\left(Z \rightarrow \ell^{+} \ell^{-}\right)$. These make the $t Z h$ process not as promising as $t Z H$ even for HL-LHC, which we make clear in the following.

To find the discovery potential, we choose a benchmark point where $A$ is heavier than $m_{h}+m_{Z}$, and lighter than $m_{H}+m_{Z}$. Such a choice would forbid $A \rightarrow Z H$ decay and enhance $\mathcal{B}(A \rightarrow Z h)$. Unlike the previous section, we also need $c_{\gamma} \neq 0$. We find such a benchmark point from $2 \mathrm{HDMC}$ that passes the perturbativity, unitarity, and positivity constraints, as well as the $T$ parameter constraint. The parameter values are $\eta_{1}=0.428, \eta_{2}=2.88, \eta_{3}=$ $0.795, \eta_{4}=2.916, \eta_{5}=2.334, \eta_{6}=-0.897, \eta_{7}=2.76$, $m_{H^{+}}=378 \mathrm{GeV}, m_{A}=401 \mathrm{GeV}, m_{H}=559 \mathrm{GeV}, c_{\gamma}=$ 0.186 , and $\mu_{22}^{2} / v^{2}=1.96$. With this set of parameters, we find that $\rho_{t c}$ values above 0.5 are excluded at $95 \%$ C.L. This is extracted from $\mathcal{B}(t \rightarrow c h)$ [35], while the constraint from the CMS CRW region [31] is a bit weaker. The branching ratios corresponding to this $\mathrm{BP}$ are $\mathcal{B}(A \rightarrow Z h) \approx 0.1$, $\mathcal{B}(A \rightarrow t \bar{c}+\bar{t} c) \approx 0.9$.

There exist several backgrounds for the $t Z h$ process. The dominant backgrounds are $t \bar{t} Z, 4 t, t \bar{t} h$, with subdominant backgrounds from $t Z+$ jets, $t \bar{t} W, 3 t+$ jets, $3 t+W$ jets, and $t W Z$. To find the discovery potential, we follow the 
same procedure to generate signal and background events as in Sec. III. We keep the signal cross section at LO, but for backgrounds we take the same QCD correction factors as in the previous section. The details of the selection cuts, and signal and background cross sections after selection cuts, are presented in an Appendix.

The statistical significance at $\sim 1.1 \sigma$ turns out to be rather small, even with the full HL-LHC data set. While significances would be lower for heavier $m_{A}$ due to a fall in the parton luminosity, it does not improve much for lighter $m_{A}$. In the latter case, i.e., for lighter $m_{A}, \mathcal{B}(A \rightarrow Z h)$ becomes lower, and the constraint on $\rho_{t c}$ becomes more stringent from the CMS CRW region [31]. For the $c g \rightarrow t A \rightarrow t Z h$ search in $h \rightarrow W^{+} W^{-*}$ and $Z \rightarrow b \bar{b}$ modes, one loses the mass reconstruction capability of $m_{Z}, m_{h}$, and $m_{A}$, hence the control of background processes. Therefore, it is likely that the $t Z h$ process would remain below sensitivity even for HL-LHC.

\section{DISCUSSION AND SUMMARY}

We have studied the discovery potential of $c g \rightarrow$ $t A \rightarrow t Z H, t Z h$ processes at the LHC. The $t Z H$ process can be discovered, albeit likely needing HL-LHC data. Discovery is possible for $m_{A} \sim 400 \mathrm{GeV}$, with statistical significance reaching up to $\sim 6 \sigma$ with the full HL-LHC data set. But $m_{A}$ cannot be much lighter or heavier than $\sim 400 \mathrm{GeV}$. The discovery prospect for the $t Z h$ process is rather limited, primarily due to the suppression from mixing angle $c_{\gamma}$ (alignment "protection"), and the constraint on $\rho_{t c}$ from $\mathcal{B}(t \rightarrow c h)$. With significance only about $1 \sigma$ at best with $3000 \mathrm{fb}^{-1}, t Z h$ seems out of reach at the LHC. We note that the $c g \rightarrow t H \rightarrow t Z A$ process is possible for $m_{H}>m_{Z}+m_{A}$, and can be searched for by a strategy similar to $t Z H$. We also remark that $\rho_{t u}$ can induce the $u g \rightarrow t A \rightarrow t Z H$ process, with similar signature. Although $\rho_{t u}$ could become stringently constrained [55], the discovery potential is balanced by large valence-quark induced $u g \rightarrow t A$ production.

In general, the presence of $\rho_{t t}$ would reduce the discovery potential of $t Z H$ because of $\mathcal{B}(A \rightarrow t \bar{t})$, but it opens up other modes for $A \rightarrow Z H$ discovery, for example, induces $A \rightarrow Z H$ signal via loop induced $g g \rightarrow A \rightarrow Z H$ [18]. The same is true for $\rho_{b b}$, where $A \rightarrow Z H$ can be induced by $g b \rightarrow b A \rightarrow b Z H$ [56] as well as $g g \rightarrow b \bar{b} A \rightarrow b \bar{b} Z H$ [56,57]. One can also have $g g \rightarrow A \rightarrow Z h$ [25] and $g g \rightarrow$ $b \bar{b} A \rightarrow b \bar{b} Z h[25,58,59]$. But both processes are again suppressed by the mixing angle $c_{\gamma}$. In general, the impact of $\rho_{b b}$ is inconsequential for the $t Z H$ process, but the presence of $\rho_{t c}$ would reduce the discovery potential for $\rho_{b b}$ induced $A \rightarrow Z H$ processes.

We have not discussed so far the uncertainties in our results. We have not included QCD correction factors for signal in both the $t Z H$ and $t Z h$ processes. In general, $c$-quark initiated processes have non-negligible systematic uncertainties such as from PDF, which we have not included in our analysis. Such uncertainties for $c$-quark initiated processes are discussed in Refs. [60,61], while a detailed discussion of PDF choices and their uncertainties for Run 2 can be found in Ref. [62]. These lead to some uncertainties in our results. A detailed estimate of such uncertainties is beyond the scope of this paper.

While the presence of $\rho_{t t}$ reduces the discovery potential of the $t Z H$ process $m_{A}>2 m_{t}$, it opens up the exquisite discovery mode $c g \rightarrow t A / t H \rightarrow t t \bar{t}$. It is also worth mentioning the "excess" seen by CMS [53] in the $g g \rightarrow A \rightarrow t \bar{t}$ search at $m_{A} \approx 400 \mathrm{GeV}$. Such excess can be interpreted within the g2HDM framework [32], if $\rho_{t t} \simeq 1.1$ and $\rho_{t c} \simeq$ 0.9 with $m_{H^{ \pm}} \gtrsim 530 \mathrm{GeV}$ and $m_{H} \gtrsim 500 \mathrm{GeV}$. Note that, for $\rho_{t t} \sim 1$, the $t Z H$ discovery (or $c g \rightarrow t H \rightarrow t Z A$ discovery) is not possible due to suppression from $\mathcal{B}(A \rightarrow t \bar{t})$ $(\mathcal{B}(H \rightarrow t \bar{t}))$ decay. However, if this excess materializes into evidence or discovery by Run $3, c g \rightarrow t A / t H \rightarrow t t \bar{c}$ might emerge immediately followed by discovery of $c g \rightarrow$ $t A / t H \rightarrow t t \bar{t}$.

In summary, motivated by electroweak baryogenesis, we analyzed the discovery potential of the $c g \rightarrow t A \rightarrow t Z H$ process. Such a process might be induced by extra Yukawa coupling $\rho_{t c}$ if one removes the discrete $Z_{2}$ symmetry from 2HDM. We find discovery is possible at the HL-LHC if $m_{A} \sim 400 \mathrm{GeV}$, but $\rho_{t t}$ would need to be small. For completeness, we have also studied the $c g \rightarrow t A \rightarrow t Z h$ process, but do not find it promising. Discovery of the $c g \rightarrow t A \rightarrow t Z H$ process will not only shed light on the strongly first order electroweak phase transition, but it may also help uncover the mechanism behind the observed baryon asymmetry of the Universe.

\section{ACKNOWLEDGMENTS}

This research is supported by MOST Grants No. 1062112-M-002-015-MY3, No. 107-2811-M-002-039, No. NTU 108L104019, and No. 108-2811-M-002-537.

\section{APPENDIX: EVENT SELECTION FOR THE $t$ Zh PROCESS}

We discuss the event selection criteria and the corresponding signal and backgrounds for the $t Z h$ process. Events are required to have at least three leptons, and at least three $b$ jets with some missing transverse energy. The $p_{T}$ of the leading and other two subleading leptons are required to be $>25,20$, and $15 \mathrm{GeV}$, respectively, with pseudorapidity $|\eta|<2.5$. The $p_{T}$ of all three $b$ jets are required to be $>20 \mathrm{GeV}$ with $|\eta|<2.5$. The $E_{T}^{\text {miss }}$ in each event should be $>35 \mathrm{GeV}$. We demand the separation $\Delta R$ between any two leptons, any two jets, and any jet and lepton to be $>0.4$. We then apply the $m_{\ell^{+} \ell^{-}}$cut: for each event there are at least two possible $m_{\ell^{+} \ell^{-}}$combinations, and the $m_{\ell^{+} \ell^{-}}$combination closest to $m_{Z}$ should be within $70 \mathrm{GeV}<m_{\ell^{+} \ell^{-}}<100 \mathrm{GeV}$. Similarly, there are at least 
two possible $m_{b b}$ combinations in each event. We demanded the one that is closest to $m_{h}$ should be within $\left|m_{b b}-m_{h}\right|<25 \mathrm{GeV}$. Finally, we construct all possible $m_{\ell \ell b b}$ combinations from the three leading leptons and leading $b$ jets, and demand the $m_{\ell \ell b b}$ combination closest to $m_{A}$ should be within $\left|m_{\ell \ell b b}-m_{A}\right|<100 \mathrm{GeV}$. The cross sections of signal and background processes after selection cuts are summarized in Table V.
TABLE V. Signal and background cross sections (in $\mathrm{fb}$ ) for the $t Z h$ process after selection cuts at $\sqrt{s}=14 \mathrm{TeV}$ LHC. The subdominant backgrounds are added together as others, and the last column is the total background.

\begin{tabular}{lccccc}
\hline \hline Signal $(\mathrm{fb})$ & $t \bar{t} Z$ & $4 t$ & $t t \bar{h}$ & Others & Total background \\
\hline 0.003 & 0.025 & 0.002 & 0.0001 & 0.0001 & 0.027 \\
\hline \hline
\end{tabular}

[1] G. Aad et al. (ATLAS Collaboration), Phys. Lett. B 716, 1 (2012); S. Chatrchyan et al. (CMS Collaboration), ibid. 716, 30 (2012).

[2] J. F. Gunion and H. E. Haber, Phys. Rev. D 67, 075019 (2003).

[3] See e.g., G. C. Branco, P. M. Ferreira, L. Lavoura, M. N. Rebelo, M. Sher, and J. P. Silva, Phys. Rep. 516, 1 (2012), and references there in.

[4] S. L. Glashow and S. Weinberg, Phys. Rev. D 15, 1958 (1977).

[5] W.-S. Hou and M. Kikuchi, Eur. Phys. Lett. 123, 11001 (2018).

[6] See also, e.g. J. Bernon, J. F. Gunion, H. E. Haber, Y. Jiang, and S. Kraml, Phys. Rev. D 92, 075004 (2015); P. Bechtle, H. E. Haber, S. Heinemeyer, O. Stål, T. Stefaniak, G. Weiglein, and L. Zeune, Eur. Phys. J. C 77, 67 (2017).

[7] K. Fuyuto, W.-S. Hou, and E. Senaha, Phys. Lett. B 776, 402 (2018).

[8] See also J. de Vries, M. Postma, J. van de Vis, and G. White, J. High Energy Phys. 01 (2018) 089.

[9] M. Kohda, T. Modak, and W.-S. Hou, Phys. Lett. B 776, 379 (2018).

[10] W.-S. Hou, M. Kohda, and T. Modak, Phys. Lett. B 786, 212 (2018).

[11] W.-S. Hou, G.-L. Lin, C.-Y. Ma, and C.-P. Yuan, Phys. Lett. B 409, 344 (1997)

[12] S. Iguro and K. Tobe, Nucl. Phys. B925, 560 (2017).

[13] Without detailed studies, the process was also discussed by W. Altmannshofer, J. Eby, S. Gori, M. Lotito, M. Martone, and D. Tuckler, Phys. Rev. D 94, 115032 (2016); W. Altmannshofer, B. Maddock, and D. Tuckler, ibid. 100, 015003 (2019); and Ref. [12]. See also S. Gori, C. Grojean, A. Juste, and A. Paul, J. High Energy Phys. 01 (2018) 108, where the $p p \rightarrow t \bar{c} H$ process was discussed.

[14] N. Turok and J. Zadrozny, Nucl. Phys. B358, 471 (1991).

[15] L. Fromme, S. J. Huber, and M. Seniuch, J. High Energy Phys. 11 (2006) 038.

[16] G. C. Dorsch, S. J. Huber, K. Mimasu, and J. M. No, Phys. Rev. Lett. 113, 211802 (2014).

[17] A. G. Cohen, D. B. Kaplan, and A. E. Nelson, Annu. Rev. Nucl. Part. Sci. 43, 27 (1993).

[18] For a nonexhaustive list, see e.g., B. Coleppa, F. Kling, and S. Su, J. High Energy Phys. 09 (2014) 161; B. Hespel, F. Maltoni, and E. Vryonidou, J. High Energy Phys. 06 (2015)
065; F. Kling, H. Li, A. Pyarelal, H. Song, and S. Su, J. High Energy Phys. 06 (2019) 031.

[19] M. Aaboud et al. (ATLAS Collaboration), Phys. Lett. B 783, 392 (2018).

[20] V. Khachatryan et al. (CMS Collaboration), Phys. Lett. B 759, 369 (2016).

[21] See, e. g., S. Davidson and H. E. Haber, Phys. Rev. D 72, 035004 (2005).

[22] A. Djouadi, Phys. Rep. 459, 1 (2008).

[23] B. Altunkaynak, W.-S. Hou, C. Kao, M. Kohda, and B. McCoy, Phys. Lett. B 751, 135 (2015).

[24] D. Chowdhury and O. Eberhardt, J. High Energy Phys. 05 (2018) 161; J. Haller, A. Hoecker, R. Kogler, K. Mönig, T. Peiffer, and J. Stelzer (Gfitter Group), Eur. Phys. J. C 78, 675 (2018); See also W.-S. Hou, M. Kohda, and T. Modak, Phys. Rev. D 98, 075007 (2018).

[25] M. Aaboud et al. (ATLAS Collaboration), J. High Energy Phys. 03 (2018) 174; A. M. Sirunyan et al. (CMS Collaboration), Eur. Phys. J. C 79, 564 (2019), and references therein.

[26] D. Eriksson, J. Rathsman, and O. Stå 1, Comput. Phys. Commun. 181, 189 (2010).

[27] M. E. Peskin and T. Takeuchi, Phys. Rev. D 46, 381 (1992).

[28] H. E. Haber and O. Stål, Eur. Phys. J. C 75, 491 (2015).

[29] C. D. Froggatt, R. G. Moorhouse, and I. G. Knowles, Phys. Rev. D 45, 2471 (1992).

[30] M. Baak and R. Kogler, arXiv:1306.0571.

[31] A. M. Sirunyan et al. (CMS Collaboration), arXiv:1908 .06463.

[32] W.-S. Hou, M. Kohda, and T. Modak, Phys. Lett. B 798, 134953 (2019).

[33] A. Crivellin, A. Kokulu, and C. Greub, Phys. Rev. D 87, 094031 (2013).

[34] S.-P. Li, X.-Q. Li, and Y.-D. Yang, Phys. Rev. D 99, 035010 (2019).

[35] M. Aaboud et al. (ATLAS Collaboration), J. High Energy Phys. 05 (2019) 123.

[36] A. M. Sirunyan et al. (CMS Collaboration), J. High Energy Phys. 06 (2018) 102.

[37] W.-S. Hou, M. Kohda, and T. Modak, Phys. Rev. D 99, 055046 (2019).

[38] J. Alwall, R. Frederix, S. Frixione, V. Hirschi, F. Maltoni, O. Mattelaer, H.-S. Shao, T. Stelzer, P. Torrielli, and M. Zaro, J. High Energy Phys. 07 (2014) 079. 
[39] R. D. Ball, V. Bertone, S. Carrazza, L. Del Debbio, S. Forte, A. Guffanti, N. P. Hartland, and J. Rojo (NNPDF Collaboration), Nucl. Phys. B877, 290 (2013).

[40] T. Sjöstrand, S. Mrenna, and P. Skands, J. High Energy Phys. 05 (2006) 026.

[41] J. de Favereau, C. Delaere, P. Demin, A. Giammanco, V. Lemaître, A. Mertens, and M. Selvaggi (DELPHES 3 Collaboration), J. High Energy Phys. 02 (2014) 057.

[42] M. L. Mangano, M. Moretti, F. Piccinini, and M. Treccani, J. High Energy Phys. 01 (2007) 013.

[43] J. Alwall et al., Eur. Phys. J. C 53, 473 (2008).

[44] A. Alloul, N. D. Christensen, C. Degrande, C. Duhr, and B. Fuks, Comput. Phys. Commun. 185, 2250 (2014).

[45] J. Campbell, R. K. Ellis, and R. Röntsch, Phys. Rev. D 87, 114006 (2013).

[46] M. Grazzini, S. Kallweit, D. Rathlev, and M. Wiesemann, Phys. Lett. B 761, 179 (2016).

[47] SM Higgs production cross sections at $\sqrt{s}=$ $14 \mathrm{TeV}$ : https://twiki.cern.ch/twiki/bin/view/LHCPhysics/ CERNYellowReportPageAt14TeV2010.

[48] J. M. Campbell and R. K. Ellis, J. High Energy Phys. 07 (2012) 052.

[49] G. Cowan, K. Cranmer, E. Gross, and O. Vitells, Eur. Phys. J. C 71, 1554 (2011).

[50] See e.g., N. Craig, F. D'Eramo, P. Draper, S. Thomas, and H. Zhang, J. High Energy Phys. 06 (2015) 137; S. Kanemura, H. Yokoya, and Y.-J. Zheng, Nucl. Phys. B898, 286 (2015); S. Gori, I.-W. Kim, N. R. Shah, and
K. M. Zurek, Phys. Rev. D 93, 075038 (2016); N. Craig, J. Hajer, Y.-Y. Li, T. Liu, and H. Zhang, J. High Energy Phys. 01 (2017) 018. These studies are for 2HDM with softlybroken $Z_{2}$ symmetry.

[51] For a recent reference, see M. Carena and Z. Liu, J. High Energy Phys. 11 (2016) 159, and references therein.

[52] M. Aaboud et al. (ATLAS Collaboration), Phys. Rev. Lett. 119, 191803 (2017).

[53] A. M. Sirunyan et al. (CMS Collaboration), arXiv: 1908.01115.

[54] K.-F. Chen (private communication); See e.g., the $m_{t j}$ resolution in excited top search, A. M. Sirunyan et al. (CMS Collaboration), Phys. Lett. B 778, 349 (2018).

[55] W.-S. Hou, M. Kohda, T. Modak, and G.-G. Wong, Phys. Lett. B 800, 135105 (2020).

[56] T. Modak, Phys. Rev. D 100, 035018 (2019).

[57] T. Modak and E. Senaha, Phys. Rev. D 99, 115022 (2019).

[58] P. M. Ferreira, S. Liebler, and J. Wittbrodt, Phys. Rev. D 97, 055008 (2018).

[59] N. M. Coyle, B. Li, and C. E. M. Wagner, Phys. Rev. D 97, 115028 (2018).

[60] M. Buza, Y. Matiounine, J. Smith, and W. L. van Neerven, Eur. Phys. J. C 1, 301 (1998).

[61] F. Maltoni, G. Ridolfi, and M. Ubiali, J. High Energy Phys. 07 (2012) 022.

[62] J. Butterworth et al., J. Phys. G 43, 023001 (2016); See also W.-S. Hou, M. Kohda, and T. Modak, Phys. Rev. D 98, 015002 (2018). 